\title{
MULTIPLE DESCRIPTION SCALABLE CODING USING WAVELET-BASED MOTION COMPENSATED TEMPORAL FILTERING
}

\author{
Mihaela van der Schaar and Deepak S. Turaga \\ Philips Research USA, Briarcliff Manor, NY 10510 \\ \{mihaela.vanderschaar, deepak.turaga\}@philips.com
}

\begin{abstract}
Packet delay jitter and loss due to network congestion pose significant challenges for designing and deploying delay sensitive multimedia applications over the best effort packet switched networks such as the Internet. Recent studies indicate that using Multiple Description Coding (MDC) in conjunction with path or server diversity can mitigate these effects. However, the proposed MDC coding and streaming techniques are based on non-scalable coding techniques. A key disadvantage of these techniques is that they can only improve the error resilience of the transmitted video, but are not able to address two other important challenges associated with the robust transmission of video over unreliable networks: adaptation to bandwidth variations and receiving device characteristics. In this paper, we present a new paradigm, referred to as Multiple Description Scalable Coding (MDSC), that is able to address all the previously mentioned challenges by combining the advantages of scalable coding and MDC. This framework enables tradeoffs between throughput, redundancy and complexity at transmission time, unlike previous non-scalable MDC schemes. Furthermore, we also propose a novel MDSC scheme based on Motion Compensated Temporal Filtering (MCTF), denominated Multiple Description Motion Compensated Temporal Filtering (MDMCTF). We use the inherent ability of current MCTF schemes, using the lifting implementation of temporal filtering. We show how tradeoffs between throughput, redundancy and complexity can easily be achieved by adaptively partitioning the video into several descriptions after MCTF. Based on our simulations using different network conditions, the proposed MD-MCTF framework outperforms existing MDC schemes over a variety of network conditions.
\end{abstract}

\section{INTRODUCTION}

Video communication over both Internet and wireless networks is becoming increasingly popular. However, reliable transmission of video over such networks poses many challenges, including coping with bandwidth variations and packet losses caused by congestion in the Internet and competing traffic, fading, interference and mobility in the wireless networks.

Recently, Multiple description coding (MDC) has been proposed in order to improve the robustness of video to packet losses. By taking advantage of the distributed infrastructure of the Internet and/or wireless networks, MDC delivers complementary descriptions from one or several video servers over multiple paths to each client. This enables various forms of diversity that lead to an improved video quality and reliability for the client, as well as an improved loadbalancing and reliability for the delivery infrastructure.

A comprehensive overview of various MDC techniques is provided by Goyal [1] A simple and efficient scheme for MDC is based on splitting the odd and even frames of a video sequence in separate, individually decodable descriptions that can be coded using "standardized" non-scalable coding schemes. Such an MDC scheme was proposed by Apostolopoulos and Wee [2], Subsequently, Wang and Lin [3] further improved this framework by designing temporal predictors that exploit not only the temporal correlations of the frames within a description, but also across the descriptions (i.e. reference frames from both descriptions are used for encoding each description). This framework is denominated Multiple Description Motion Compensation (MDMC).

The previously mentioned MDC schemes, as well as a majority of the MDC coding techniques proposed so far, are build on top of a nonscalable motion-compensated prediction framework. An advantage of this approach is that existing standardized, high-efficiency codecs could be employed for the implementation of the new MDC schemes. However, a shortcoming of these techniques is that they are aimed solely at increasing the robustness to link congestion or link outage as well as server failure, and do not address other important transmission challenges, such as bandwidth variations, provided Quality-of-Service, device heterogeneity.

These other important challenges are often addressed by employing scalable coding. However, while neither MDC nor scalable coding can independently address all of the issues related to the efficient transmission of video over such networks, they can complement each other to provide an efficient solution. Subsequently, we refer to the combination of MDC and scalable coding as Multiple Description Scalable Coding (MDSC). A brief description of the MDSC framework, the provided flexibility and properties is given in this paper.

One example of a MDSC scheme was proposed by Bajic and Woods [4] and it is denominated domain-based MDC. In domain-based MDC, wavelet coefficients are partitioned into maximally separated sets, and packetized so that simple error concealment methods can produce good estimates of lost data. However, this scheme is mainly targeted towards image transmission, and the extensions to video are not efficient because they do not consider spatio-temporal correlations.

In this paper, we discuss the advantages of MDSC schemes that allow adjusting the amount of redundancy and the bit-rate allocated to each description at transmission time, based on the channel conditions. After describing several MDSC schemes, we introduce an efficient MDSC embodiment - multiple description motion compensated temporal filtering (MD-MCTF) - that is based on recently developed 3D interframe wavelet coding techniques. Improved temporal filtering techniques and entropy coding, have led to these 3D wavelet coding schemes being extremely efficient while providing truly scalable (temporal, SNR spatial, and complexity) bitstreams. True scalability allows easy adaptation to variations in available bandwidth and receiver capabilities and preferences. The proposed MDSC scheme is based on the Motion Compensated Temporal Filtering (MCTF) introduced by $\mathrm{Ohm}$ [5] and later improved by Choi and Woods [6]. We partition the scalable and very efficient bitstreams obtained using MCTF-based wavelet coding into two or more substreams that are sent over independent transmission channels. If some of these sub-streams are lost, we use the inherent ability of MCTF schemes, using the lifting implementation of temporal filtering, to recover missing data. We evaluate the performance of our proposed MD-MCTF scheme using simple network simulations.

This paper is organized as follows. We discuss the MDSC paradigm, its merits and several embodiments in Section2. In Section 3, after a 
brief introduction of MCTF, we describe the proposed MD-MCTF structure. We present results under different network scenarios and include brief comparisons with MDMC and domain-based MCTF in Section 4. We conclude with a summary and future research directions in Section 5.

\section{MULTIPLE DESCRIPTION SCALABLE CODING}

As mentioned above, both MDC and scalable coding and streaming were proposed for transmission over unreliable IP networks. However, if the channel characteristics change dynamically or across the network as the stream traverses different types of links (wired or wireless), one or the other method can become inefficient and not robust. Also, it should be noted that the various proxies have only limited processing capabilities and hence, full encoding-decoding solutions are not possible. Thus, for efficient end-to-end transmission over a variety of wired and wireless IP networks, a unified and flexible coding and streaming strategy is required that can easily adapt to the channel conditions, while requiring that the video data is coded just once for all these various network characteristics.

For this, we introduce the concept of MDSC that enables the mapping of a scalable video stream into several multiple description scalable video streams without transcoding and vice-versa, the mapping of multiple description scalable video streams into a single scalable coded bitstream. Using the MDSC concept, we can easily split a bitstream that was already coded in a scalable format into two or more descriptions, i.e. independently decodable bitstreams. In MDSC, the number and composition of transmitted scalable descriptions is adapted on-the-fly based on the channel conditions experienced by the various paths, application requirements (delay etc.), etc. Also, the transmission bit-rate of each description can easily be adapted.

Several embodiments of such MDSC schemes are:

- FGS-based MDSC, where the base-layer of the FGS coded video is transmitted in all descriptions, while the enhancementlayer frames are split into several descriptions.

- Forward Error Correction (FEC) based MDSC techniques as introduced in [7], where the various layers of a scalable coded bitstreams are split across the several descriptions, while also adding unequal FEC to enable improved recovery of specific layers based on their importance.

- Multiple description motion compensated temporal filtering that is introduced in the next section.

To switch between a single description scalable coded stream and multiple description scalable coding streams and vice-versa, we re- map the various packets into different priority levels. For scalable coding, the base-layer packets are transmitted using the highest priority, while the various enhancement-layer are sent using lowerpriorities. For MDSC, the various base-layer and enhancement-layer packets are distributed across multiple descriptions of equal importance. Note that for MDSC, the throughput is defined based on the number of layers in each description, while the protection level is based on the redundancy level used for each description. Alternatively, in scalable coding, the number of transmitted priority layers is determined based on the available throughput and the protection level used for each layer. The real-time re-mapping between scalable coding and MDSC and vice-versa is performed adaptively by taking into consideration the priorities of the various packets, the channel conditions, application requirements (delay etc.). This can be easily illustrated using a simple example in Figure 1 for the FEC-based MDC.

\section{MULTIPLE DESCRIPTION MOTION COMPENSATED TEMPORAL FILTERING}

\subsection{Brief review of MCTF}

MCTF is aimed at removing the temporal redundancies of video sequences. Unlike predictive coding schemes, MCTF does not employ a temporal recursive structure. Instead it uses a pyramidal decomposition to remove, very efficiently, both long-term and shortterm temporal dependencies. During MCTF, frames are filtered temporally in the direction of motion, prior to performing the spatial transformation and coding, as illustrated in Figure 2 Video frames are filtered into L (low frequency or average) and $\mathrm{H}$ (high frequency or difference) frames. This filtering is performed in the direction of motion and can be written using the lifting formulation [8], [9] as follows:

$H(y, x)=c_{2}\left(B(y, x)-A\left(y+v_{y}, x+v_{x}\right)\right)$,

$L(y, x)=c_{3}\left(2 c_{2} A(y, x)+H\left(y-v_{y}, x-v_{x}\right)\right)$,

where $\left(v_{y}, v_{x}\right)$ is the motion vector and $c_{1}, c_{2}$ are constants that may be chosen to make this filtering process orthonormal. Also shown in the figure is the pyramidal decomposition structure. At the second stage, the L frames are decomposed further into LL and LH frames, i.e. average of average frames and difference of average frames. Such a lifting based implementation allows the use of multiple reference frames and bi-directional filtering. Moreover, nondyadic decomposition structures can also easily be implemented.

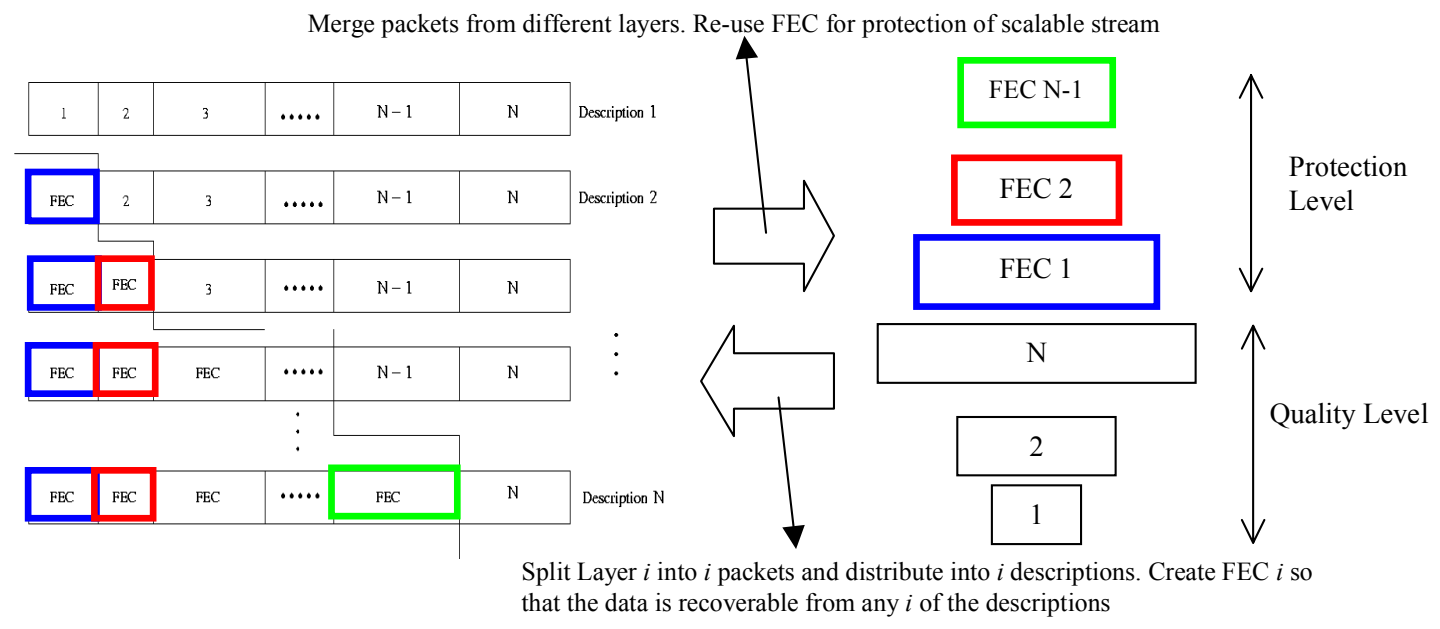

Figure 1. Re-mapping between FEC-based MDSC and single description scalable coding 


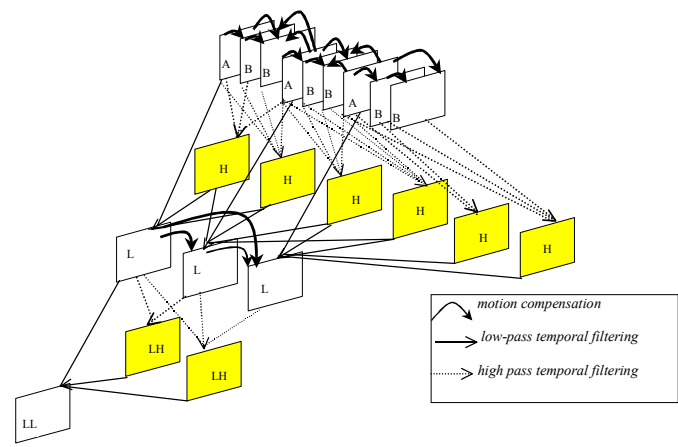

Figure 2. Example MCTF structure using Lifting

\subsection{MD-MCTF}

As described in the previous section, $\mathrm{L}$ frames are temporally filtered averages of a set of two or more consecutive frames. Thus, the $\mathrm{L}$ frames along with the motion information can be successfully used to create approximations for this consecutive set of frames. In the example of Figure 2 we can see that given the LL frame, and the motion vector information, we can create approximations for the three L frames, without using the two residue LH frames. Further, using these approximate $\mathrm{L}$ frames, we can then create approximations for all original $\mathrm{A}$ and $\mathrm{B}$ frames.

Based on the above observation, we can conclude that when splitting an MCTF-coded bitstream in two or more descriptions transmitted over independent paths, as long as the L-frame(s) obtained in the last stage of temporal decomposition is part of all descriptions, reasonable video quality can be obtained even if only one description is correctly received. The lost frames can be obtained using approximations obtained using the L-frames and the corresponding motion-vectors or approximations of these motion vectors. Of course, the received video quality improves as the number of correctly received descriptions increases. As discussed in Section 2, the specific remapping (splitting) strategy is determined based on the bandwidth and reliability of the various transmission paths. One mechanism for splitting the MCTF-based scalable bitstream adopted in this paper is illustrated in Figure 3 for the previous example of a group of 9 frames.

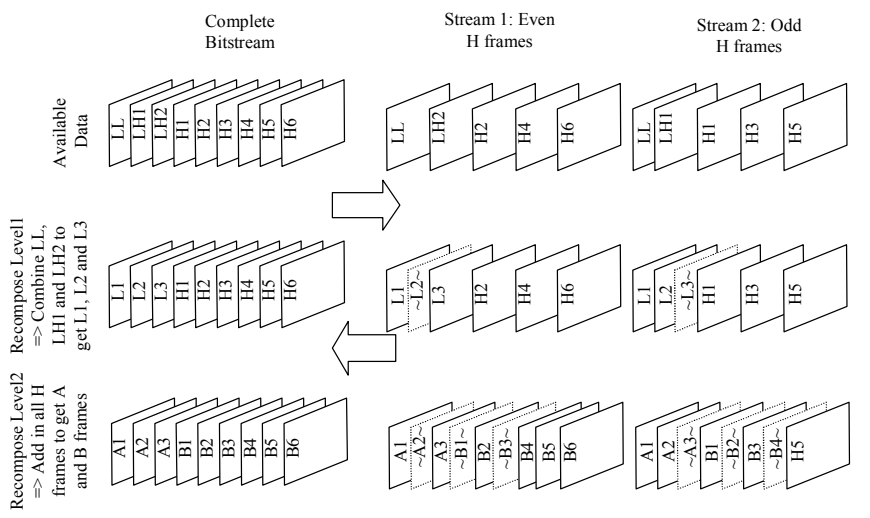

\section{Figure 3. Re-mapping of scalable MCTF into MD-MCTF}

As shown in the figure, the scalable bitstream can be re-mapped into two separate descriptions: one containing the odd $\mathrm{H}$ frames, and the other containing the even $\mathrm{H}$ frames. When the complete bitstream is received, by simply inverting the MCTF procedure, the original frames from the received LL frame, two LH frames and $6 \mathrm{H}$ frames can be obtained. However, when some frames are lost, we can approximate them using the correctly received $\mathrm{L}$ frames and the motion vector information. Hence, during recovery at Level 1 for Stream 1, we can use LL and the motion information to create an approximation for frame L2, that we label as $\sim \mathrm{L} 2 \sim$, and represent by a dotted frame. Similarly we can also create the approximate frame $\sim \mathrm{L} 3 \sim$, for Stream 2 . We may then use these approximately recovered frames to recover the other frames.

Of course, when we split the original into these two streams, we send some duplicate or redundant information. This includes the motion vectors $\amalg_{\text {and }}$ the first frame LL. Hence the overall redundancy comes from transmitting 1 extra frame per 9 frames, and one additional set of motion vectors. For instance, at $100 \mathrm{Kbps}$, this corresponds to a redundancy of approximately $15 \%$. Similarly, we can also transmit in both streams, frames LL, LH1 and LH2, and then split the remaining $\mathrm{H}$ frames into even and odd. We can also further increase the redundant information being transmitted. With increasing redundancy, the number of frames that we need to approximate is reduced, thereby increasing the decoded video quality, however this comes at an increased cost in terms of the amount of information that needs to be transmitted. Furthermore, depending on the channel conditions experienced over the independent paths, the bit-rate of the various scalable description will be adjusted correspondingly.

\section{RESULTS AND DISCUSSION}

To test the performance of the MD-MCTF, we examine two different scenarios. The first scenario consists of sending the two streams over separate channels, with one stream completely lost while the other is completely received. We use two sequences for this test, Foreman and Akiyo. Both sequences are QCIF $(176 \times 144)$ at $30 \mathrm{~Hz}$ and have medium and low amounts of motion respectively. We show in Figure 4 the redundancy rate distortion curves for these sequences for different bit rates (depicted in the figure in Kbps). All these results are generated by re-mapping a single scalable bitstream using different splitting strategies (different redundancy levels) and transmitting them at various bit-rates.

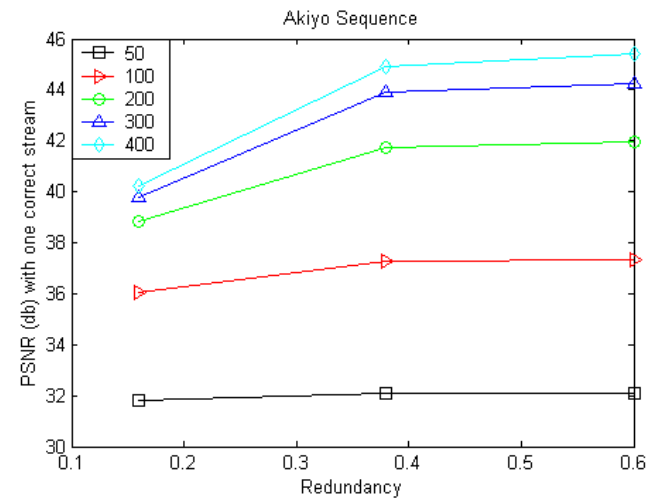

Figure 4. Redundancy Rate Distortion curves (Akiyo)

\footnotetext{
${ }^{1}$ Note that not all the motion vectors need to be transmitted in each description. Only the motion vectors used in the coarsest level of the temporal pyramid need to be available in all descriptions. If lost, the other motion vectors could be then interpolated using this motion vectors. However, if these motion vectors are interpolated, the quality of the concealed frames will be lower.
} 


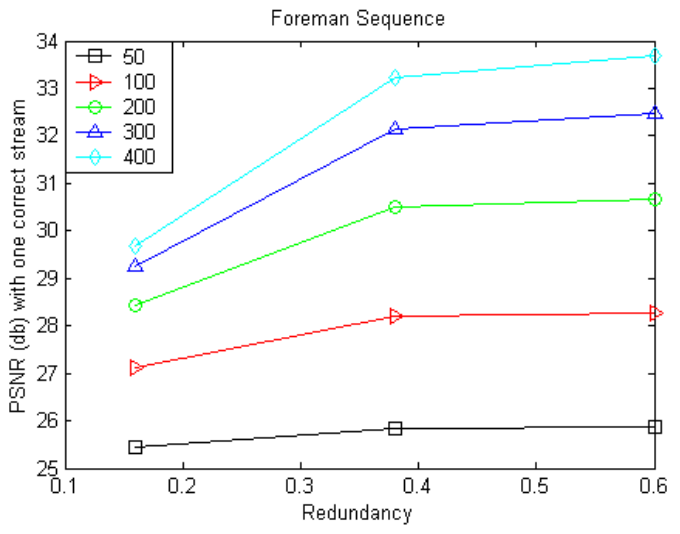

Figure 5. Redundancy Rate Distortion curves (Foreman)

As may be seen from the curves, the PSNR of the received stream increases with the amount of redundancy, however it tends to saturate after 0.38 . We also see that the performance for the Akiyo sequence is much better than for the Foreman sequence, due to its low motion, thereby allowing very good approximations of missing frames. We repeat these experiments for multiple bit rates, 50, 100, 200, 300 and $400 \mathrm{Kbps}$, to emphasize that MD-MCTF produces streams that can easily adapt to varying network conditions, and all these results are plotted as separate curves. Wang and Lin [3] also presented MDMC results for these sequences using a fixed quantization step size corresponding to a bit rate of roughly 200-300 Kbps. We may compare with our results at these bit rates and conclude that MDMCTF outperforms MDMC on the average by around 3-4 $\mathrm{dB}$ for the Akiyo sequence and 1.5-2 $\mathrm{dB}$ for the Foreman sequence. These results were obtained for MD-MCTF, even though no postprocessing or error concealment was used in the MD-MCTF decoder. The second scenario we consider is when we send the two streams over the same channel, but with packet losses. We simulate the channel using a bursty loss model, using a two-state Markov chain as proposed by Yajnik et al [10]. We consider that each video frame is packetized into a fixed number of packets, with the loss of any one of them resulting in the loss of the entire frame. For our experiments, we use three packets per frame. As before, we repeat the experiment for different amounts of redundancy and bit rates. We also compare our results to those presented by Bajic and Woods [4] for domain based MDC. We set the loss parameters to a loss rate of $15 \%$ and a burst size of 2 and use the Mobile sequence at size CIF $(352 \times 288)$ at 30 $\mathrm{Hz}$, to duplicate their experiment. The redundancy rate distortion curves are plotted in Figure 5.

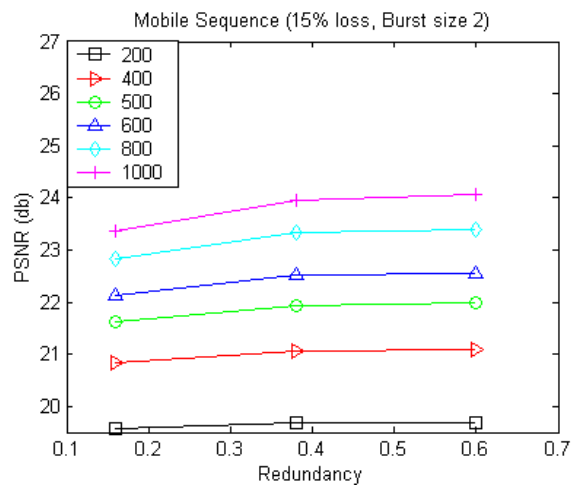

Figure 6. Redundancy Rate distortion curves

As before, the PSNR increases with redundancy, however it does not increase significantly after 0.35 . We can compare our results at 500 Kbps with those presented for the domain based MDC. The PSNR reported by Bajic and Woods, with a packet loss of $12.4 \%$ and a burst size of 2 for the same sequence was $19.1 \mathrm{~dB}$. Bajic and Woods do not provide the redundancy required for their scheme, hence we compare our results using the lowest redundancy, and find that we still outperform domain based MDC by around 2-3 dB.

\section{CONCLUSION}

In this paper we introduce a new MDSC scheme called MD-MCTF. This scheme is used in conjunction with wavelet based video coding schemes, thereby providing truly scalable and highly error resilient video transmission over lossy wireless networks. MD-MCTF enables adjusting the splitting strategy, amount of redundancy and the bit-rate allocated to each description at transmission time, while using the same pre-encoded scalable stream. Furthermore, we include some preliminary quality comparisons with previously proposed MDC schemes and conclude that MD-MCTF outperforms them by around 1.5-4 dB in PSNR, for different sequences, at multiple bit rates, and under different test conditions. The adaptation to network conditions and receiver capabilities can be easily performed at different proxies in the network since it involves only selecting a re-mapping strategy (no transcoding necessary). Directions for future work include combining the scheme with better error concealment strategies, using more accurate network test conditions, and further modifying the lifting scheme for enhanced robustness.

\section{REFERENCES}

[1] V. K Goyal, "Multiple Description Coding: Compression meets the network, " IEEE Signal Processing Magazine, vol. 18, no. 5, pp. 74-93, Sept. 2001.

[2] J.G. Apostolopoulos and S.J.Wee, "Unbalanced Multiple Description Video Communication Using Path Diversity", International Conference on Image Processing (ICIP), October 2001.

[3] Y. Wang and S. Lin, "Error resilient video coding using multiple description motion compensation," IEEE Trans. Circuits and Systems for Video Technology, vol. 12, no. 6, pp. 438-52, June 2002.

[4] I. V. Bajic and J. W. Woods, "Domain-based multiple description coding of images and video," Proc. SPIE, vol. 4671 (Visual Commun. Image Proc. 2002), pp. 124-135, San Jose, CA, January 2002.

[5] J. R. Ohm, "Three-dimensional subband coding with motion compensation," IEEE Trans. Image Proc., vol. 3, no. 5, September 1994.

[6] S.-J. Choi and J. W. Woods, "Motion compensated 3-D subband coding of video," IEEE Trans. Image Proc., vol. 8, no. 2, February 1999.

[7] R. Puri, K.-W. Lee, K. Ramchandran, V. Bharghavan, "Forward Error Correction (FEC) Codes based Multiple Description Coding for Internet Video Streaming and Multicast”, Signal Processing: Image Communication, vol. 16, no. 8, pp. 745-762, May 2001.

[8] A. Secker and D. Taubman, "Motion-compensated highly scalable video compression using an adaptive $3 \mathrm{D}$ wavelet transform based on lifting”, Proc. ICIP 2001, Thessaloniki, Oct. 2001.

[9] B. Pesquet-Popescu and V. Bottreau, "Three-dimensional lifting schemes for motion compensated video compression", Proc. ICASSP 2001, Salt Lake City, May 2001.

[10] M. Yajnik, S. Moon, J. Kurose, D. Towsley, "Measurement and modeling of the temporal dependence in packet loss," Proc. IEEE INFOCOM, pp. 345-52, March 1999. 